\title{
Randomised trial of elective continuous positive airway pressure (CPAP) compared with rescue CPAP after extubation
}

\author{
N J Robertson, P A Hamilton
}

\begin{abstract}
Aim-To determine if a weaning regimen on flow driver continuous positive airway pressure (CPAP) would decrease the number of ventilator days but increase the number of CPAP days when compared with a rescue regimen.

Methods-Fifty eight babies of 24-32 weeks gestation with respiratory distress syndrome (RDS) were studied prospectively. After extubation they were randomly allocated to receive CPAP for 72 hours $(n=29)$ according to a weaning regimen, or were placed in headbox oxygen and received CPAP only if preset "start CPAP" criteria were met $(n=29$, rescue group).

Results-There was no difference in successful extubation at 72 hours, 1 and 2 weeks, between the groups in terms of the number of reventilation episodes, reventilation days, or in total days of CPAP. Birthweight, gestational age, race, day of first extubation, antenatal or postnatal steroids, patent ductus arteriosus status and maximal mean airway pressure used were of no value in predicting success or failure at 72 hours, 1 , or 2 weeks.

Conclusion-The weaning regimen did not decrease the number of ventilator days or days on CPAP compared with the rescue regimen. The rescue regimen on flow driver CPAP seems to be a safe and effective method of managing a baby of 24-32 weeks gestation who has been ventilated for RDS or immature lung disease. (Arch Dis Child Fetal Neonatal Ed 1998;79:F58-F60)
\end{abstract}

Keywords: flow driver continuous positive airway pressure; extubation; respiratory distress syndrome; weaning

Prolonged use of mechanical ventilation has been associated with infections, chronic lung disease, ${ }^{1}$ subglottic stenosis, ${ }^{2}$ and aspiration. ${ }^{3}$ Considerable effort is therefore directed towards early weaning from the ventilator.

Over the past 25 years many devices have been used to administer CPAP. The twin nasal cannula was first described in $1973,{ }^{45}$ and was developed further in 1988 into "flow driver CPAP." ${ }^{6}$ This device generates CPAP in the immediate vicinity of the nasal airway by converting kinetic energy from a jet of fresh gas. Compared with the traditional CPAP system using standard nasal prongs, the airway pressure variations and external workload were considerably less with flow driver CPAP. ${ }^{6}$
There is no clear evidence that CPAP facilitates extubation in infants weighing less than $1500 \mathrm{~g}$ at birth. So et $\mathrm{al}^{7}$ found that nasal CPAP facilitated successful extubation in babies under $1500 \mathrm{~g}$ of birthweight. Higgins et al ${ }^{8}$ also found that nasal CPAP facilitated extubation in infants $<1000 \mathrm{~g}$ of birthweight. However, Chan and Greenough ${ }^{9}$ found no difference in extubation success between nasal CPAP or headbox oxygen in infants with acute or chronic lung disease. Likewise, Annibale et al ${ }^{10}$ found that nasopharyngeal CPAP did not improve the likelihood of successful extubation in babies $<1500 \mathrm{~g}$ birthweight in the first 2 weeks of life. Tapia et al ${ }^{11}$ also found no difference in extubation outcome in babies extubated to CPAP or headbox oxygen, although this study included a group who had pre-extubation endotracheal CPAP which worsened extubation outcome. ${ }^{12}$

Flow driver CPAP has been used in our neonatal unit for the past 3 to 4 years to give extra support after extubation of a neonate $<1500 \mathrm{~g}$ birthweight. A graded weaning programme consisting of gradually decreasing hours of flow driver CPAP and increasing hours of headbox oxygen was adopted but had not been validated. It was our clinical impression that the flow driver reduced the time spent on the ventilator but that the rigid weaning programme increased the time spent on respiratory support.

\section{Methods}

Babies who met the following criteria were entered into the trial: gestational age 24-32 weeks; ventilated for RDS or immature lung disease; duration of ventilation $>24$ hours and $<28$ days; pre-set extubation criteria; informed parental consent.

Babies were excluded if they had major congenital malformation; birth asphyxia resulting in encephalopathy; accidental or unplanned extubation; confirmed sepsis.

Ethical approval for the study was obtained. The trial was discussed with the parents early on in the clinical course. Where parental consent was obtained, the baby was randomly allocated immediately before extubation to receive either the weaning or rescue programme. The randomisation, obtained by random number allocation, was revealed by opening numbered, sealed envelopes. Babies were stratified according to gestational age-above or below 28 weeks. Babies were considered to be suitable for extubation if they were loaded with caffeine, were not clinically septic, and 
Table 1 Baseline characteristics for categorical variables

\begin{tabular}{|c|c|c|c|}
\hline & Rescue $(n=29)$ & Weaning $(n=29)$ & \\
\hline Characteristic & $n(\%)$ & $n(\%)$ & $p$ Value $^{\star}$ \\
\hline$<28$ weeks gestation & $20(69 \%)$ & $19(66 \%)$ & 1.0 \\
\hline Female & $12(41 \%)$ & $11(38 \%)$ & 1.0 \\
\hline Caucasian & $20(69 \%)$ & $21(72 \%)$ & 1.0 \\
\hline Antenatal steroids & $22(76 \%)$ & $24(83 \%)$ & 0.7 \\
\hline Postnatal steroids & $7(24 \%)$ & $3(10 \%)$ & 0.3 \\
\hline Surfactant & $29(100 \%)$ & $28(97 \%)$ & 1.0 \\
\hline PDA closed & $20(69 \%)$ & $22(76 \%)$ & 0.8 \\
\hline HFOV & $8(28 \%)$ & $8(28 \%)$ & 1.0 \\
\hline
\end{tabular}

^Fisher's exact test.

Table 2 Baseline characteristics for continuous variables

\begin{tabular}{lcccc}
\hline & \multicolumn{2}{c}{ Rescue $(n=29)$} & & \multicolumn{2}{c}{ Weaning $(n=29)$} & \\
\cline { 2 - 2 } Variable & Median (range) & & Median (range) & p Value \\
\hline Gestational age (weeks) & $27(25-30)$ & & $27(24-32)$ & 0.21 \\
Birthweight (g) & $960(598-1300)$ & & $1018(632-1680)$ & 0.11 \\
Day of 1st extubation & $4(1-26)$ & & $5(1.5-2.6)$ & 0.6 \\
Max MAP (cm $\left.\mathrm{H}_{2} \mathrm{O}\right)$ & $12(8.7-26.4)$ & & $14(7.8-30)$ & 0.7 \\
\hline
\end{tabular}

* two sample $t$ test.

$\mathrm{MAP}=$ mean airway pressure.

Table 3 Outcome for categorical variables

\begin{tabular}{|c|c|c|c|}
\hline & Rescue $(n=29)$ & Weaning $(n=29)$ & \\
\hline Outcome & $n(\%)$ & $n(\%)$ & $p$ Value ${ }^{\star}$ \\
\hline Extubated at 72 hours & $22(76 \%)$ & $23(79 \%)$ & 1.0 \\
\hline Extubated at 1 week & $26(90 \%)$ & $23(79 \%)$ & 0.5 \\
\hline Extubated at 2 weeks & $26(90 \%)$ & $26(90 \%)$ & 1.0 \\
\hline Death & $1(3 \%)$ & $5(17 \%)$ & 0.1 \\
\hline Intraventricular haemorrhage (grade 1-4) & $4(14 \%)$ & $6(21 \%)$ & 0.7 \\
\hline Retinopathy of prematurity (max grade 2 ) & $1(3 \%)$ & $2(7 \%)$ & 0.6 \\
\hline $\mathrm{O}_{2}$ dependency at 36 weeks & $10(34 \%)$ & $15(52 \%)$ & 0.3 \\
\hline \multicolumn{4}{|l|}{ Number of reventilation episodes } \\
\hline 0 & $19(66 \%)$ & $18(62 \%)$ & 0.6 \\
\hline 1 & $7(24 \%)$ & $8(28 \%)$ & \\
\hline 2 & $0(0 \%)$ & $1(3 \%)$ & \\
\hline 3 & $3(10 \%)$ & $1(3 \%)$ & \\
\hline
\end{tabular}

${ }^{\star}$ Fisher's exact test. headbox. Regular blood gases were checked at 2-4 hourly intervals. Should the blood gases deteriorate to "start CPAP" criteria the baby was placed on flow driver CPAP at a pressure of $6 \mathrm{~cm} \mathrm{H}_{2} \mathrm{O}$ for 12 hours. If the blood gases were normal at the end of this period the baby was then replaced in a head box and regular blood gases checked again. If rescued more than three times, the baby was then deemed to have "failed on rescue" and was then weaned off flow driver CPAP, as described in the weaning programme.

The start CPAP criteria were $\mathrm{p} \mathrm{H}<7.27$ or FIO2 $>0.6$, or frequent bradycardias/apnoeas

Reventilation criteria were $\mathrm{p} \mathrm{H}<7.2$ or FIO2 $>0.9$, or profound desaturation, or two $\mathrm{p} \mathrm{H}$ values $<7.25$.

If reventilation criteria were met, the baby was reintubated and ventilated until extubation was considered clinically appropriate. The baby was then extubated on to the same protocol as initial randomisation. This design was chosen instead of a cross over design so as to be able to measure the total number of flow driver and reventilation days for each baby and to assess potential longer term complications.

Success was defined as remaining extubated for 72 hours after the first extubation. The sample size was calculated from looking retrospectively at 17 babies weaning off CPAP to assess the standard error of the mean of time to wean. The regression of log wean time on gestational age was highly significant and we therefore stratified the groups into $24-27$ and 28-32 completed weeks. We looked at reduction in time to wean of $50 \%$. The sample size required with a power of $90 \%$; p value $5 \%$ to detect a $50 \%$ reduction in time to wean was 29 in each group.

For each categorical variable, Fisher's exact test was used to compare the two groups and for each continuous variable, $t$ tests were used. All continuous variables except gestational age were $\log$ transformed in order to achieve equality of variances and normality of residuals.

\section{Results}

Sixty one babies were considered to be eligible for entry into the trial. Three babies were subsequently excluded because of entry before receiving 24 hours of ventilation. There was no significant difference between groups in baseline characteristics at trial entry (tables 1 and 2).

There was no significant difference between the groups for the following: success at extubation at 72 hours, 1 and 2 weeks, death, intraventricular haemorrhage, retinopathy of prematurity, oxygen dependence at 36 weeks and total number of reventilation episodes (table 3).

There was no significant difference between the two groups for total days of reventilation after initial extubation and total CPAP days (table 4).

Logistic regression analysis was used to predict success or failure of extubation at 72 hours, 1 or 2 weeks. The following baseline characteristics were analysed: birthweight; 
Table 4 Outcome for continuous variables

\begin{tabular}{|c|c|c|c|}
\hline & Rescue $(n=29)$ & Weaning $(n=29)$ & \\
\hline Outcome & Mean $(95 \% C I)$ & Mean $(95 \% C I)$ & $p$ Value $^{\star}$ \\
\hline Total days reventilation after extubation & $1.0(0.3-1.9)$ & $0.9(0.3-1.8)$ & 0.9 \\
\hline Total days CPAP & $5.0(2.5-9.3)$ & $9.0(5.7-14.0)$ & 0.13 \\
\hline
\end{tabular}

*two sample $t$ test.

gestation; race; day of first extubation; antenatal steroids; use of postnatal steroids; patent ductus arteriosus status and maximal mean airway pressure. In no case were any of the odds ratios significantly $(p<0.05)$ different from 1-they had no predictive value for success or failure of extubation at 72 hours, 1 and 2 weeks.

Logistic regression analysis was then used to predict oxygen dependency at 36 weeks. Late first extubation, high mean airway pressure, low gestational age, lack of antenatal steroids and caucasian race were predictive of oxygen dependency at 36 weeks $(\mathrm{p}<0.05)$.

\section{Discussion}

It was our hypothesis that those babies, especially those of less than $1000 \mathrm{~g}$ birthweight, who were randomised to the rescue regimen would develop atelectasis and require longer time on the ventilator than those weaned off flow driver CPAP more slowly on the weaning regimen. It was also felt that those randomised to the rescue regimen might require reventilation more often than those gradually weaned off CPAP. This was not the case.

Babies randomised to the weaning regimen spent a longer time on flow driver CPAP than those randomised to the rescue regimen, although this did not reach significance. The most common reason for requiring rescue CPAP or failure to wean on CPAP was respiratory acidosis. Apnoeas, bradycardias, and increasing oxygen requirements above $60 \%$ were also common causes of requirement for CPAP. Similarly, the most common reason for reventilation was respiratory acidosis. Three babies required reventilation because of culture positive sepsis - one baby in the rescue group and two babies in the weaning group. There were slightly more deaths in the weaning group than in the rescue group, but these deaths were due to sepsis and were not related to the weaning method of CPAP.

There was no significant difference between the two groups in successful extubation at 1 or 2 weeks. One week after extubation there were slightly more babies on CPAP $(41 \%)$ in the rescue group than in the weaning group $(27 \%)$.
Two weeks after extubation there was little difference between groups, with $34 \%$ of the rescue group and $45 \%$ of the weaning group being on CPAP.

The failure rate of extubation at 72 hours was $24 \%$ in the rescue group and $21 \%$ in the weaning group. Overall, the failure rate of extubation was 33\% in Greenough's study and $40 \%$ in Annibale's study. These figures are slightly higher than the rate of extubation failure in our study (21-24\%). This could be explained by the fact that flow driver CPAP is better tolerated by babies than the "long prong," or by the fact that no baby was deprived of flow driver CPAP if it was clinically indicated - we did not feel it was appropriate to have a control group who did not receive CPAP at all. Those babies developing respiratory acidosis, reaching "start CPAP" criteria improved when put on to CPAP.

As far as we are aware, this is the first study in babies to date using the flow driver CPAP device after extubation. It is also the first study to look at different regimens of CPAP which can be used during the recovery stage of RDS. Our findings show that it is safe to extubate into headbox oxygen as long as the baby is monitored regularly and flow driver CPAP is given if required.

1 Avery ME, Tooley WH, Keller JB, et al. Is chronic lung disease in low birthweight infants preventable? A survey of eight centres. Pediatrics 1987;79:26-30.

2 Fan LL, Flynn JW, Pathak DR. Risk factors predicting laryngeal injury in intubated neonates. Crit Care Med laryngeal injury

3 Goodwin SR, Graves SA, Haberkern CM. Aspiration in intubated preterm infants. Pediatrics 1985;75:85-8.

4 Agostino R, Orzalesi M, Nodari S, et al. Continuous positive airway pressure by nasal cannula in the respiratory distress syndrome of the newborn. Pediatr Res 1973;7:50

5 Kattwinkel J, Fleming D, Cha CC, Klaus MH. A device for administration of continuous positive airway pressure by the nasal route. Pediatrics 1973;52:131-4.

6 Moa G, Nilsson K, Zetterstrom H, Jonsson L. A new device for administration of nasal continuous positive airway pressure in the newborn: an experimental study. Crit Care Med 1988;16;1238-42.

7 So BH, Tamura M, Mishina J, Watanabe T, Kamoshita S. Application of nasal continuous positive airway pressure to early extubation in very low birthweight infants. Arch Dis Child 1995;72:F191-3.

8 Higgins RD, Richter SE, Davis JM. Nasal continuous airway pressure facilitates extubation of very low birthweight neonates. Pediatrics 1991;88:999-1003.

9 Chan V, Greenough A. Randomised trial of methods of extubation in acute and chronic respiratory distress. Arch Dis Child 1993;68:570-2.

10 Annibale DJ, Husley TC, Engstrom PC, Wallin LA, Ohning BL. Randomised controlled trial of nasopharyngeal continuous positive airway pressure in the extubation of very low birthweight infants. F Pediatr 1994:124:455-60.

11 Tapia contin, Bancali A, Gonzalez A, Mercado ME. Does continuous positive airway pressure during weaning from intermittent mandatory ventilation in very low birthweight infants have risks or ben

12 Kim EH, Boutwell WC. Successful direct extubation of very low birthweight infants from low intermittent mandatory ventilation rate. Pediatrics 1987;80:409-14. 